\title{
FenOlOGía, CRECIMIENTO EN DIÁMETRO Y PERIODICIDAD DE Hura polyandra en Costa Grande, Guerrero, México
}

\author{
Elizandro Pineda-Herrera ${ }^{1,5}$, Juan Ignacio Valdez-Hernández ${ }^{2}$, \\ Carmen de la Paz Pérez-Olvera ${ }^{3}$ y Raymundo Dávalos-Sotelo ${ }^{4}$ \\ ${ }^{1}$ Doctorado en Ciencias Biológicas y de la Salud, Universidad Autónoma Metropolitana \\ ${ }^{2}$ Postgrado Forestal, Colegio de Postgraduados, campus Montecillo, Edo. Mex. \\ ${ }^{3}$ Laboratorio de Anatomía de la Madera. Universidad Autónoma Metropolitana Unidad Iztapalapa \\ ${ }^{4}$ Red de Ambiente y Sustentabilidad, Instituto de Ecología, A.C. Xalapa, Ver. \\ ${ }^{5}$ Autor para la correspondencia: elherrera2001@yahoo.com.mx.
}

\begin{abstract}
Resumen: Se evaluó la fenología de Hura polyandra Baill. en una selva mediana subcaducifolia de Zihuatanejo de Azueta, Guerrero, registrando bimestralmente durante dos años: caída de hojas, floración, fructificación y brote de hojas, correlacionando estas variables con la precipitación y la temperatura. Se relacionó el diámetro de árboles con la fenología. Se midió el crecimiento en diámetro cada dos meses durante dos años, empleando bandas dendrométricas estableciendo seis categorías diamétricas, tres simetrías y tres iluminaciones de la copa y ajustando los incrementos a un modelo matemático. La periodicidad del crecimiento se determinó realizando marcajes al cambium de un árbol a 1.30 m (DAP) cada dos meses y obteniendo la rodaja correspondiente al término del experimento para determinar si los anillos formados con anuales. H. polyandra se defolia de marzo a mayo, florece de marzo a julio, fructifica de marzo a septiembre y su brote de hojas ocurre de julio a enero. Se registraron correlaciones significativas entre el brote de hojas con la precipitación $(P \leq 0.01)$ y la floración con la temperatura $(P \leq 0.01)$. El crecimiento fue mayor y significativo $(P \leq 0.001)$ en las categorías diamétricas 10,15 y $20 \mathrm{~cm}$, la iluminación de copas favoreció de forma significativa $(P \leq 0.01)$ incrementos mayores en diámetro. En la zona de estudio, esta especie forma un anillo de crecimiento anual.
\end{abstract}

Palabras clave: Bandas dendrométricas, iluminación de copa, selva mediana subcaducifolia, simetría de copa, Zihuatanejo de Azueta.

\begin{abstract}
Phenology Hura polyandra Baill. was evaluated in a semideciduous tropical forest of Azueta Zihuatanejo, Guerrero, recording bimonthly for two years: falling leaves, flowering, fruiting and leaf sprout, correlating these variables with precipitation and temperature. Diameter trees were associated with phenology. Using dendrometer bands growth diameter was measured every two months for two years, establishing six diameter categories, three symmetries and three crown lighting, adjusting the growth increments to a mathematical model. Growth periodicity was determined by performing markings on the cambium of a tree at 1.30 $\mathrm{m}(\mathrm{DBH})$ every two months and obtaining the corresponding slice at the end of the experiment to determine whether annual rings were formed. Hura polyandra defoliates from March to May, flowers from March to July, bear fruits from March to September and sprouts the leaves from July to January. Significant correlations occurred between the development of leaves buds with precipitation $(P \leq 0.01)$ and flowering with temperature $(P \leq 0.01)$. The growth is higher and significant $(P \leq 0.001)$ in diameter categories 10,15 and $20 \mathrm{~cm}$. Crown lighting helps significantly $(P \leq 0.01)$ greater increases in diameter. In the study area, this species forms an annual ring.
\end{abstract}

Keywords: Dendrometer bands, crown lighting, tropical semideciduos forest, crown symmety, Zihuatanejo de Azueta.

$\mathbf{E}$ n México, la distribución de las selvas secas ha sido amplia, ya que se les encuentra en toda la vertiente del pacífico, la península de Yucatán, la cuenca del Balsas, Sur de Tamaulipas y la parte central de Veracruz (Miranda y Hernández-X., 1963; Rzedowski, 1978). Representan un gran acervo biológico, considerando que en conjunto tanto sel- vas caducifolias, subcaducifolias y espinosas comprenden aproximadamente el $40 \%$ de especies de plantas vasculares endémicas del país (Rzedowski, 1991).

Una de las características más conspicuas que poseen las selvas secas es la estacionalidad de la precipitación (hasta ocho meses secos), que destaca como el principal factor que 
dirige la ocurrencia y la dinámica de aspectos como la fenología, la regeneración, el establecimiento y el crecimiento de las especies vegetales (Bullock y Solis-Magallanes, 1990; Ceccon et al., 2006). La temporada de lluvias, su intensidad y periodos de sequía intermedios también participan en la contracción-aumento del diámetro a través de la inducción de la actividad cambial, lo cual se relaciona con los eventos foliares y reproductivos; traduciéndose en la periódica formación de anillos de crecimiento anual (Worbes 1999).

Investigaciones fenológicas realizadas en selvas secas de Australia (Myers et al., 1998), Bolivia (Justiniano y Fredericksen, 2000), Brasil (Lisi et al., 2008), Costa Rica (Borchert, 1994) y Sudáfrica (Shackleton, 2002) adjudican a la precipitación y al almacenamiento de agua edáfica los patrones encontrados. Otras investigaciones realizadas en Australia (Williams et al., 1997), Brasil (Marques et al., 2004), India (Murali y Sukumar, 1994) y Kenia (Kinnaird, 1992) se correlacionan de forma contundente con la temperatura y factores bióticos.

En Bolivia (López et al., 2012), Brasil (Rossato, 2009; Mendivelso et al., 2014), Costa Rica (Chapman y Chapman, 1990), Ghana (Baker et al., 2002), India (Chaturvedi et al., 2011) y Sudáfrica (Shackleton, 2002) se encontró que el incremento en diámetro anual está estrechamente relacionado con el inicio, duración y cantidad de lluvia, status hídrico de los fustes, así como por la cantidad y calidad de luz.

Baker et al. (2003) destacan que especies pioneras tropicales son altamente demandantes de luz para crecer, algo común en selvas húmedas. Sin embargo aún existen interrogantes adicionales acerca del incremento en árboles de distintas edades y del papel de la calidad-integridad de copa en árboles de selvas secas (Sokpon y Biaou, 2002).

La información del crecimiento en diámetro es importante para conocer la dinámica sucesional, establecer turnos de corta maderable y se complementa con el conocimiento de la edad de los árboles de interés, obteniéndola p. ejemplo a través del análisis de los anillos de crecimiento. Esta herramienta es esencial para determinar la periodicidad con que crece el fuste y registrar la dinámica del clima u otros factores (Worbes, 1999).

En México, la fenología de selvas secas se ha estudiado principalmente en la selva baja caducifolia de Chamela, Jalisco en los trabajos de: Bullock y Solis-Magallanes (1990), Bullock (2002), Lobo et al. (2003), Borchert et al. (2004), Herrerias-Diego et al. (2006), Hayden et al. (2010) y Méndez-Alonso et al. (2013). En estas investigaciones se encontró que la precipitación, seguida por: el uso diferencial del agua entre especies, la temperatura, el fotoperiodo y factores endógenos configuran los patrones fenológicos de las especies. En Yucatán Valdez et al. (2010) mencionan que la fenología se relaciona totalmente con el uso diferencial del agua de cada especie y a la heterogeneidad edáfica.

Los estudios de crecimiento en selvas de México (Whigham et al., 1990; Bullock, 1997) y que incluyan aspectos de fenología y de anillos de crecimiento (López-Ayala et al., 2006a, 2006b; Yañez-Espinosa et al., 2006; Galán-Larrea et al., 2011; Makocki et al., 2012) son incipientes y en muchas regiones se carece de conocimiento que derive en una comprensión esencial de su dinámica fenológica-dendrológica y en un manejo silvícola sostenible (Makocki et al., 2012).

Una de las especies pioneras representativas de las selvas medianas subcaducifolias mexicanas es Hura polyandra Baill. (Habillo, Haba de San Ignacio, Jabilla) (Euphorbiaceae), la cual se caracteriza por poseer pronunciadas espinas cónicas en su tronco y una forma particular de dispersión: sus frutos dehiscentes al llegar a la madurez, estallan para propagar sus semillas (Pennington y Sarukhán, 2005). Es valorada por sus atributos maderables (Silva, 2008; Román et al., 2011; Pineda-Herrera et al., 2012c), como forraje (hojas secas) para el ganado vacuno (Román et al., 2011), alimento (frutos inmaduros) para fauna silvestre (Fam. Psittacidae) (CONABIO, 2011) y en la medicina tradicional (corteza y semillas) (Flores y Ricalde, 1996).

En 1996 Gallardo realizó una descripción florística del parque ecológico "La Vainilla" en el municipio de Zihuatanejo de Azueta, Guerrero, en el cual registró la floración durante seis meses de una muestra de árboles de la selva mediana subcaducifolia. La autora menciona a Hura polyandra en la relación de especies evaluadas, aunque no específica la duración exacta de la evento, otras fenofases o su relación con factores bióticos o abióticos. En una investigación reciente en Cosalá, Sinaloa, Estrada-Castelo et al. (2012) mencionan que la fenología foliar de $H$. polyandra, está influida por la humedad del suelo.

En Costa Grande, Guerrero, la región geopolítica más extensa a nivel estatal $\left(14710.70 \mathrm{~km}^{2}\right)$, se encuentran poblaciones de Hura polyandra en selvas medianas subcaducifolias que forman parte de la Sierra Madre del Sur (Pineda-Herrera et al., 2012a), catalogada como prioritaria por su biodiversidad y servicios ambientales (Mittermeier et al., 1999). Dada la importancia cultural, ecológica y económica de la especie, resulta esencial estudiar su fenología, tasa de crecimiento en diámetro y edad de individuos creciendo en condiciones naturales.

Considerando lo anterior, se plantearon las siguientes preguntas de investigación: 1) ¿la estacionalidad de la precipitación y las oscilaciones de la temperatura condicionan la fenología foliar y reproductiva de Hura polyandra en Costa Grande, Guerrero? 2) ¿el tamaño de los árboles influye en la intensidad de los eventos fenológicos? 3) ¿diferentes categorías diamétricas y distintas condiciones de copa influyen en el incremento en diámetro? 4) ¿considerando la estacionalidad de la precipitación, se formará un solo anillo anual de crecimiento?

Los objetivos fueron: 1) evaluar la caída y el brote de hojas, la floración y la fructificación de Hura polyandra durante dos años y correlacionarlos con la precipitación y temperatura, 2) relacionar las diferentes categorías diamétricas 
Fenología, crecimiento en diámetro y periodicidad de Hura polyandra en Costa Grande, Guerrero, México.

con los eventos fenológicos, 3) medir durante dos años el crecimiento en diámetro para establecer diferencias entre categorías diamétricas, simetrías e iluminaciones de copa y 4) establecer la periodicidad de formación de sus anillos de crecimiento.

\section{Materiales y Métodos}

Área de estudio. La localidad "El Chilcahuite" (400 m s.n.m.) en el ejido "La Laja y anexos" $\left(17^{\circ} 53^{\prime} 55^{\prime}\right.$ " N, $100^{\circ}$ 42' 44' W) (INEGI, 2005), en el municipio de Zihuatanejo de Azueta que se ubica en Costa grande, Guerrero (Figura 1). Fisiográficamente pertenece a la provincia Sierra Madre del Sur y a la subprovincia Cordillera Costera del Sur. Geomorfológicamente El Chilcahuite es una cañada con pendientes pronunciadas y lomeríos moderados con afloramientos de rocas ígneas extrusivas y sedimentarias (Meza y García, 1997). El suelo es de origen volcánico de textura migajón-arcillosa (Meza y García, 1997). El clima es cálido subhúmedo con lluvias en verano (Aw): con una precipitación total anual que oscila entre 1,100 a 1,500 milímetros temperatura media anual 16 a $28^{\circ} \mathrm{C}$ (Meza y García, 1997) (Figura 1).

Es una selva mediana subcaducifolia sin manejo silvícola y con antecedentes de pastoreo vacuno caracterizada

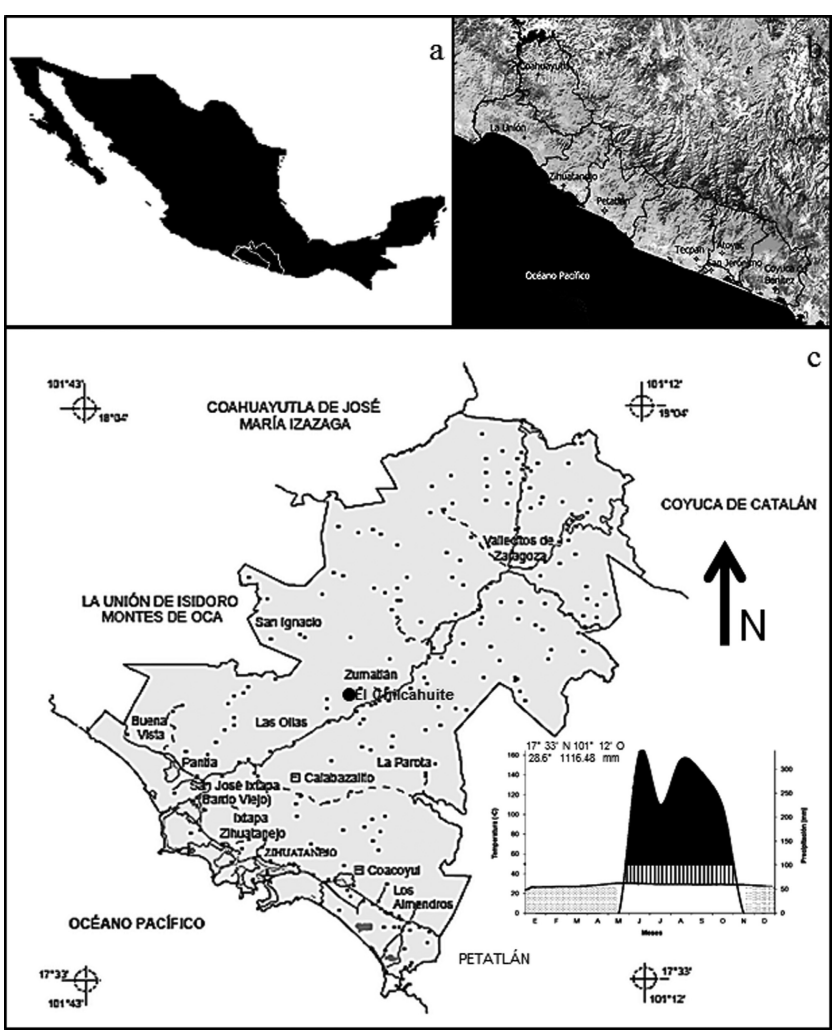

Figura 1. Zona de estudio A. Estado de Guerrero. B. Costa Grande, Guerrero. C. El Chilcahuite. por géneros como: Brosimum, Bursera, Cedrela, Ceiba y Hura. La altura promedio es mayor a los 15 metros, en general de 25 a $30 \mathrm{~m}$, con algunos ejemplares emergentes que superan los $30 \mathrm{~m}$ (Brosimum alicastrum) (Pineda-Herrera et al., 2012a).

Fenología. Se identificaron por orden de aparición en la selva 16 individuos sanos con diámetros de 25 a $70 \mathrm{~cm}$, de fuste recto y en edad reproductiva de acuerdo a lo recomendado por Fournier-O y Charpantier (1975). Bimestralmente desde mayo de 2010 a Julio de 2012 se registró utilizando binoculares en cada uno de los 16 individuos: a) caída de hojas, b) flores, c) frutos y d) brote de hojas; todos expresados en una escala del cero al cuatro según el porcentaje de presencia de cada evento: Ausencia total del evento (0), $1-25 \%$ (1), 26 $-50 \%$ (2), $51-75 \%$ (3) y $76-100 \%$ (4) (Pineda-Herrera et al., 2012b). Los valores promediados de todos los árboles para cada evento por bimestre se correlacionaron (Spearman) (Gotelli y Ellison, 2004) con la temperatura y precipitación, obtenidos para los periodos de observación de la estación Vallecitos de Zaragoza del Servicio Meteorológico Nacional $(\mathrm{SMN}, 2012)\left(17^{\circ} 55^{\prime} 20^{\prime} \mathrm{N}, 101^{\circ} 19^{\prime} 02^{\prime \prime} \mathrm{O}\right)$ que se localiza a $20 \mathrm{~km}$ de distancia de la localidad. El programa de estadística utilizado fue STATA (STATA Corp., 2001).

Con el propósito de establecer la relación entre diámetro $(\mathrm{DAP}=1.30 \mathrm{~m}$ del suelo) de los 16 árboles y la fenología, se categorizaron en seis intervalos: $22.5-27.49 \mathrm{~cm}$ (categoría 25); 27.5 - $32.49 \mathrm{~cm}$ (categoría 30), 32.5 - 42.49 (categoría 40), 42.5 - $52.49 \mathrm{~cm}$ (categoría 50), 52.5 - 62.49 $\mathrm{cm}$ (categoría 60) y $62.5-72.49 \mathrm{~cm}$ (categoría 70 ). Se promediaron los valores para cada evento y se graficaron de acuerdo a su diámetro para el periodo de observación mayo 2010 - julio 2012.

Crecimiento en diámetro. Se midió el diámetro de 21 árboles encontrados por orden de aparición en la selva, distintos a los elegidos para el estudio de fenología. Bimestralmente desde Mayo del 2010 hasta Julio del 2012 se midieron empleando bandas dendrométricas (López-Ayala et al., 2006a; Makocki et al., 2012). Los árboles se clasificaron en seis categorías diamétricas (tres árboles mínimo por cada categoría): 2.5 - $7.49 \mathrm{~cm}$ (categoría 5), $7.5-12.49 \mathrm{~cm}$ (categoría 10), $12.5-17.49 \mathrm{~cm}$ (categoría 15), $17.7-22.49 \mathrm{~cm}$ (categoría 20), $22.5-27.49 \mathrm{~cm}$ (categoría 25 ) y $27.5-32.49 \mathrm{~cm}$ (categoría 30).

Las categorías se definieron por un límite inferior y uno superior de medición, determinado por la disponibilidad de individuos. Estos límites se establecen con el propósito de obtener la dinámica de crecimiento por estructura de edades de la población a estudiar (Melo-Cúz y Vargas-Ríos, 2003).

Las copas de los árboles medidos de ambas especies se categorizaron modificando la propuesta de Dawkins (1958): Simetría o calidad de copa. 1: copa asimétrica, degradada, dañada, con una o pocas ramas 2: copa parcialmente simétri- 
ca o rala, con medio circulo o medio ovalo 3: copa simétrica, formando un circulo completo, perfecto. Iluminación de copa. 1: recepción de luz vertical o lateral poca, enteramente sombreada 2: recepción vertical o lateral parcial, sombreada parcialmente por otras copas 3: recepción vertical o lateral total, la parte superior de la copa totalmente expuesta a la luz y libre de competencia lateral.

Los incrementos por categoría y condición de copa se ajustaron al modelo no lineal Gompertz (ecuación 1).

$$
y=y_{\infty} \times \exp \left(-\exp \left(-g \times\left(t-t_{0}\right)\right)\right)
$$

donde:

$y=$ Variable de estudio (diámetro)

$y_{\infty}=$ Parámetro de valor asintótico

$g$ = Parámetro de significado biológico limitado

$t_{0}=$ Parámetro que determina la tendencia de la curva en el tiempo

$t=$ Edad (días)

La modelación de los incrementos se realizó en el paquete STATA en el módulo de análisis de regresión no lineal (StataCorp, 2001). La comparación estadística entre las categorías diamétricas y las condiciones de copa en el paquete PAC (Programas para el análisis de crecimiento) (Salgado-Ugarte et al., 2005) mediante la prueba de razón de verosimilitud (Kimura, 1980) que calcula un estadístico que es comparado con la distribución $\chi^{2}$ con grados de libertad iguales al número de restricciones, de acuerdo a la siguiente ecuación

$$
\chi_{k}^{2}=-N \times L_{\mathrm{n}}\left[\frac{S C R_{\Omega}}{S C R_{\text {世 }}}\right]
$$

donde: $k$ son los grados de libertad, $N$ es el número total de observaciones combinado de las dos curvas que se están comparando, $S C R_{\Omega}$ es la suma total de cuadrados de los residuos obtenida de ajustar ambas curvas por separado y $S C R_{\omega}$ es la suma total de residuos cuadrados derivados de ajustar a las curvas con algunas de las restricciones supuestas ( $Y$ s iguales, $g s$ iguales, $t$ s iguales). La pareja de curvas con diferencias estadísticas significativas presentarán valores más altos de razón de verosimilitud ( $R V)$ (Salgado-Ugarte et al., 2005).

Periodicidad. Se eligió un individuo de categoría diamétrica $15 \mathrm{~cm}$. Varias investigaciones en otras selvas han señalado la factibilidad de marcar pocos individuos, siempre y cuando formen parte de la comunidad investigada y se hagan estudios complementarios como la fenología, el crecimiento radial o en altura (Detienne, 1989; 1995). Detienne (1995) recomienda que para una identificación confiable de anillos sean elegidos árboles jóvenes con la finalidad de disminuir la probabilidad de encontrar anillos falsos o discontinuos.

$\mathrm{Al}$ árbol elegido se le realizaron incisiones de aproximadamente $3 \mathrm{~mm}$ de ancho, $5 \mathrm{~mm}$ de profundidad y $100 \mathrm{~mm}$ de longitud a 1.30 m (DAP) cada dos meses de Mayo de 2010 a Marzo de 2011, bimestralmente de septiembre de 2011 a enero de 2012, en abril y julio de 2012. En esta última visita, el árbol se derribó para obtener la rodaja correspondiente y determinar si el crecimiento fue anual (López-Ayala et al., 2006b; Galán-Larrea et al., 2011).

Para observar microscópicamente las estructuras celulares que delimitaron los anillos de crecimiento, la rodaja se sumergió en agua destilada durante 48 horas para ablandarla. De su cara transversal se obtuvieron cortes de aproximadamente $20 \mu \mathrm{m}$ de grosor con una cuchilla de micrótomo, se tiñeron con fucsina básica al 0,05\% durante 30 segundos y después en azul astra al 0,5\% durante dos minutos. Se deshidrataron en una serie gradual de alcoholes del $50 \%, 70 \%$, $85 \%, 96 \%$ y $100 \%$. Después de deshidratados se colocaron en xilol y se montaron con resina sintética (modificado de Sandoval, 2005).

\section{Resultados}

Fenología. La caída de hojas de Hura polyandra se observó en mayo de 2010, de noviembre de 2010 a mayo de 2011 y de enero a abril de 2012 se repitió este patrón. La floración abarcó las visitas de mayo a julio, en los dos primeros años de evaluación y en las últimas dos evaluaciones de 2012. Los frutos se presentaron de mayo a julio de 2010, mayo a septiembre de 2011 y abril a julio de 2012, el brote de hojas de agosto de 2010 a marzo de 2011 y de junio de 2011 a enero de 2012 (Figura 2).

La caída de hojas fue el evento más conspicuo de los observados, ya que en la mayoría de los individuos en más de un $90 \%$ carecieron de follaje en las visitas entre marzo y mayo. Durante la época de floración se observaron flores masculinas y femeninas simultáneamente. Los frutos observados en el estudio son estructuras evidentes y reconocibles por su tamaño y estructura. El brote de hojas es un evento que se caracteriza por las dimensiones de la lámina foliar $(25 \times 15 \mathrm{~cm})$ (Figura 3).

La figura 4 muestra que la caída de hojas tiene sus mayores picos en la época seca, con el aumento de la temperatura, de forma inversa los mayores valores del brote de hojas coinciden con la temporada de precipitación y cierta disminución de la temperatura. En el caso de la floración fue evidente su presencia antes de las lluvias, con las mayores temperaturas del año principalmente en la primavera de 2011. La fructificación se presentó además en época de secas (2010) y simultáneamente en época de lluvias (2011 y 2012).

\begin{tabular}{llllllllllllll}
\multicolumn{1}{c}{ Años } & \multicolumn{3}{c}{2010} & \multicolumn{1}{c}{} & \multicolumn{1}{c}{2011} & & & \multicolumn{2}{c}{2012} \\
\hline Eventos/Meses & M & J & S & N & E & M & M & J & S & N & E & A & J \\
\hline Caída de Hojas & & & & & & & & & & & & & \\
Floración & & & & & & & & & & & & & \\
Fructificación & & & & & & & & & & & & & \\
Brote de Hojas & & & & & & & & & & & & & \\
\hline
\end{tabular}

Figura 2. Calendario fenológico de Hura polyandra. 


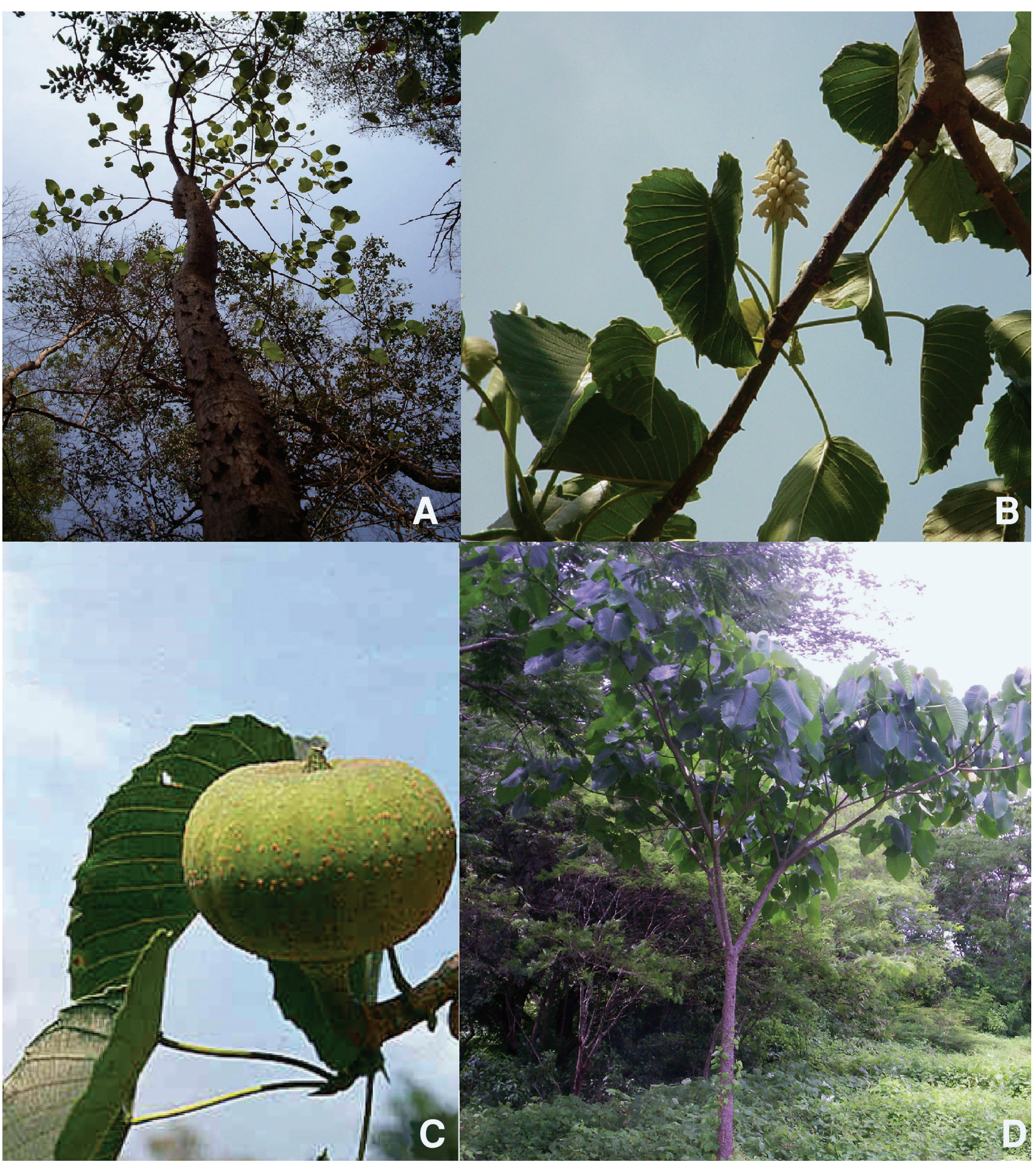

Figura 3. Eventos fenológicos de Hura polyandra. A) Caída de hojas. B) Floración. C) Fructificación. D) Brote de hojas.

La caída de hojas se correlacionó de forma inversa y significativa $(P \leq 0.01)$ con la precipitación y la floración lo hizo de forma positiva y significativa con la temperatura $(P$ $\leq 0.01)$. La floración, fructificación y el brote de hojas no se asociaron con la precipitación. La fenología foliar y la fructificación tampoco se correlacionaron con la temperatura (Tabla 1).

La relación intensidad fenológica vs categorías diamétricas mostró que diámetros de $25 \mathrm{~cm}$ tienen un mayor vigor para el brote de hojas aunque se defolian más, los diámetros 

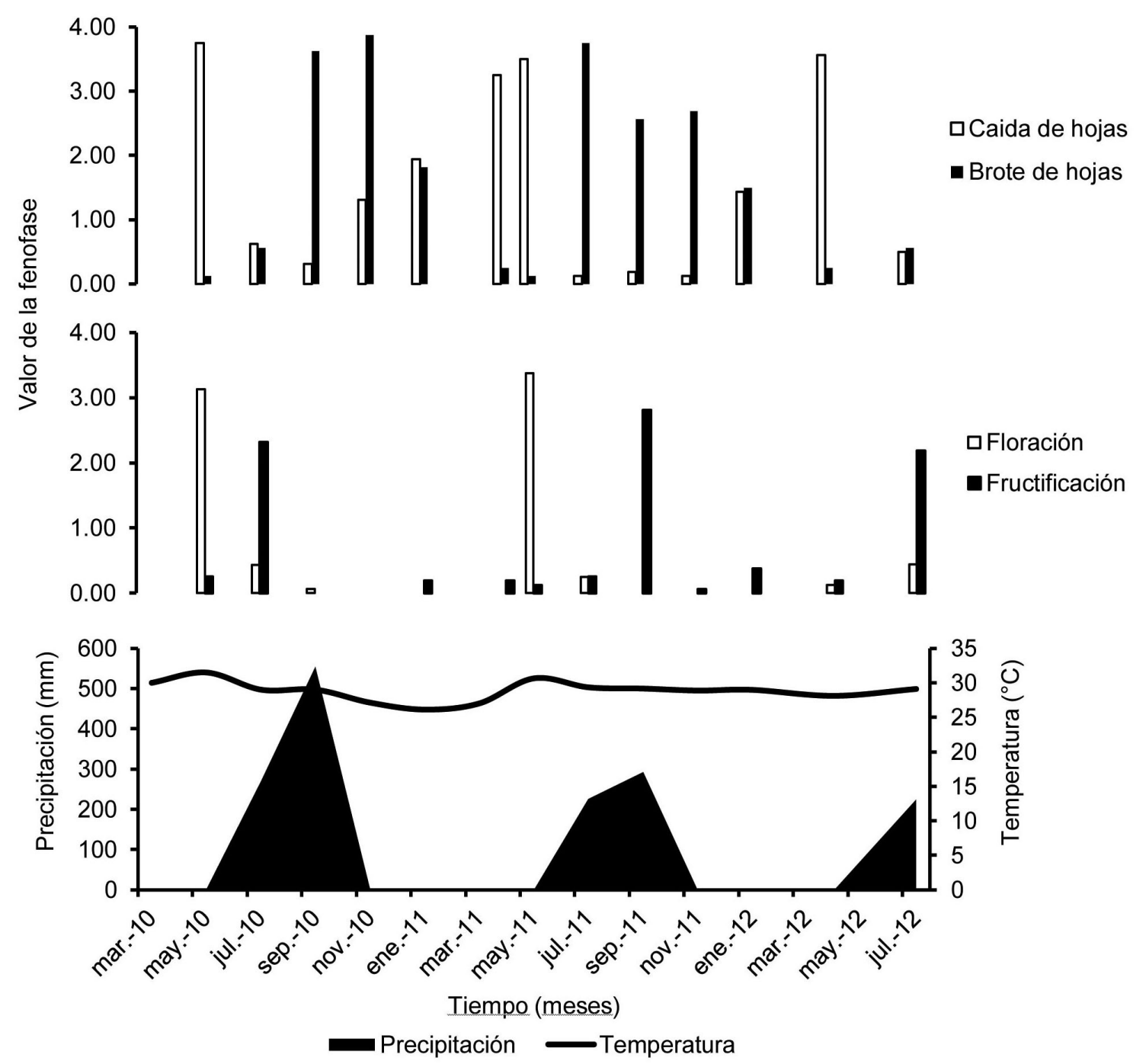

Figura 4. Dendrofenograma de Hura polyandra.

mayores presentaron una mayor intensidad reproductiva (Figura 5).

Crecimiento en diámetro. El ajuste al modelo de crecimiento mostró altos valores estadísticos y significativos $(P \leq$ 0.01). Los árboles crecieron más en la época de lluvias del segundo año de medición, principalmente durante los meses de agosto a octubre (Figura 6).

Los ajustes para cada categoría diamétrica mostraron $r^{2}$

Tabla. 1. Correlación paramétrica entre variables climáticas y eventos fenológicos.

\begin{tabular}{lcccc}
\hline Variable & $\begin{array}{c}\text { Caída de } \\
\text { Hojas }\end{array}$ & Floración & Fructificación & $\begin{array}{c}\text { Brote } \\
\text { de Hojas }\end{array}$ \\
\hline Precipitación & $-0.6352^{*}$ & 0.1534 & 0.3601 & 0.3774 \\
Temperatura & -0.0882 & $0.7318^{*}$ & 0.3703 & -0.2445 \\
\hline
\end{tabular}

${ }^{*} p<0.01$ altas y significancia estadística $(P \leq 0.01)$ coincidiendo los mayores incrementos con los meses de lluvia (junio a octubre). (Figura 7).

La tabla 2 muestra los incrementos anuales promedio por categoría diamétrica, $15 \mathrm{~cm}$ presenta el mayor valor seguida por $10,5,25$ y $30 \mathrm{~cm}$. El incremento máximo lo tuvo la categoría 10 y el mínimo de toda la muestra correspondió a la categoría $25 \mathrm{~cm}$.

La prueba estadística comprobó las diferencias de la tabla 2, al encontrar significancia $(P \leq 0.01)$ entre las categorías 10 y 25,10 y 30,15 y 25,15 y 30,20 y 25,20 y 30; dichas interacciones fueron las que presentaron mayores valores de razón de verosimilitud ( $R V$ ) (Tabla 3 ).

Las curvas para las condiciones de copa mostraron un patron similar entre aquellos parcialmente simétricos y totalmente simétricos y hubo mayor incremento en los árboles totalmente iluminados respecto a los parcialmente iluminados (Figura 8). 


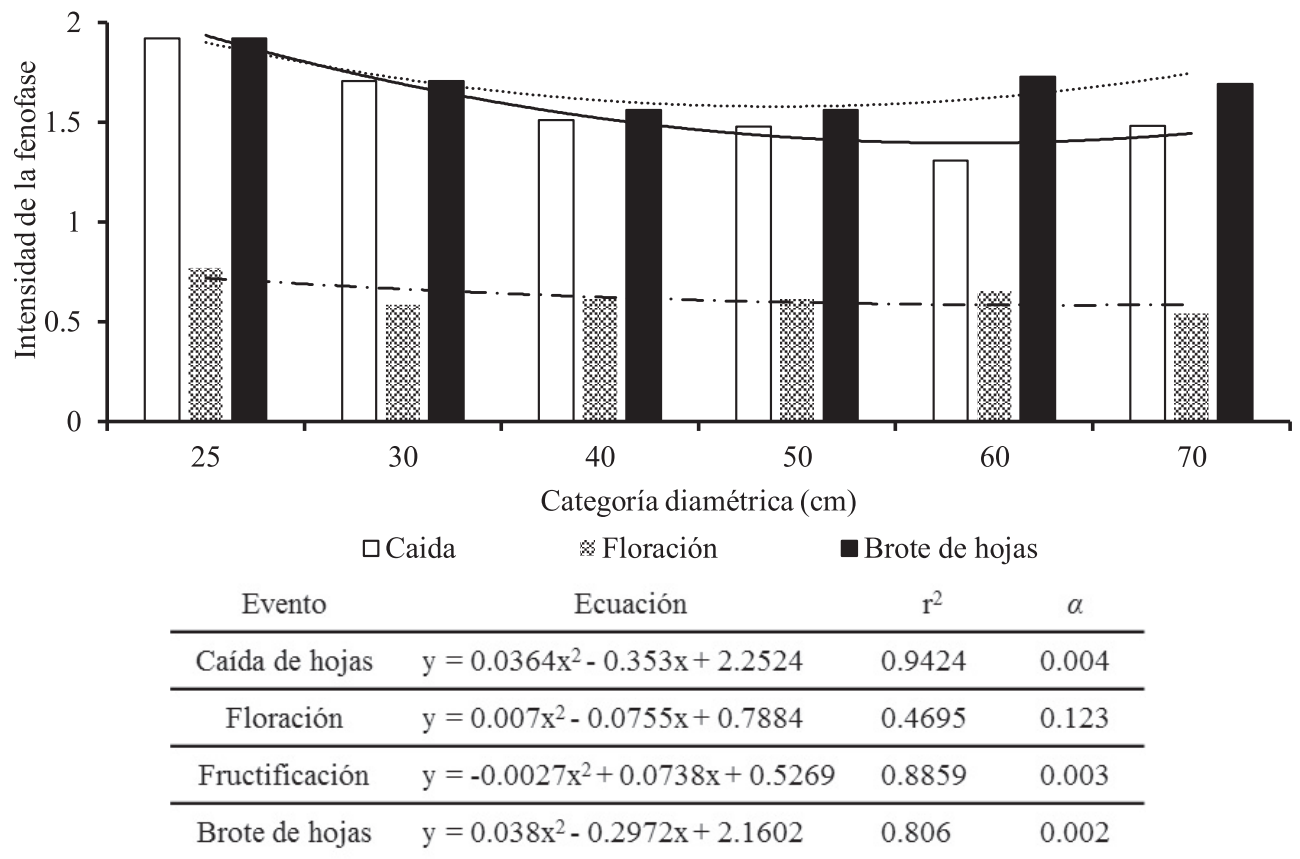

Figura 5. Relación fenológica vs. categorías diámetricas de Hura polyandra

Los incrementos de los árboles de acuerdo a su simetría fueron mayores en árboles con simetría parcial que en árboles simétricos y mayores en aquellos con copas totalmente iluminadas (Tabla 4). El análisis de verosimilitud mostró que no hubo diferencia significativa entre simetrías 2 y 3 , pero la iluminación total favoreció de forma significativa $(P$ $\leq 0.01$ ) el crecimiento en diámetro (Tabla 5).

Periodicidad. Los anillos de crecimiento fueron visibles, anuales y están delimitados por una o dos hileras de fibras compactas radialmente, así como por hasta cinco bandas de parénquima. Se aprecian células de paredes gruesas y diámetros pequeños en la franja que delimita el anillo entre periodos de crecimiento y células de paredes delgadas y lúmenes amplios en el periodo de crecimiento. No se detectaron anillos falsos o discontinuos. La porosidad es difusa. (Figura 9).

Tabla 2. Crecimiento en diámetro anual de Hura polyandra.

\begin{tabular}{lccccc}
\hline $\begin{array}{l}\text { Categoría } \\
\text { diamétrica } \\
(\mathrm{cm})\end{array}$ & $\begin{array}{c}\text { Número } \\
\text { de } \\
\text { árboles }\end{array}$ & \multicolumn{2}{c}{$\begin{array}{c}\text { Crecimiento en diámetro } \\
\text { (cm/año) }\end{array}$} & $\begin{array}{c}\text { Desviación } \\
\text { Estándar }\end{array}$ \\
\cline { 3 - 5 } 5 & 3 & 0.13 & 0.24 & 0.32 & 0.12 \\
10 & 4 & 0.15 & 0.42 & 0.69 & 0.28 \\
15 & 4 & 0.25 & 0.45 & 0.63 & 0.24 \\
20 & 3 & 0.19 & 0.32 & 0.43 & 0.16 \\
25 & 4 & 0.12 & 0.17 & 0.28 & 0.09 \\
30 & 3 & 0.13 & 0.14 & 0.18 & 0.06 \\
Todas & 21 & 0.16 & 0.29 & 0.42 & 0.20 \\
\hline
\end{tabular}

\section{Discusión}

La caída de hojas de Hura polyandra durante los meses de mayor sequía en esta investigación (marzo-mayo) coincidió con lo estudiado por Estrada-Castelo et al. (2012) para la misma especie en Sinaloa, México (altitud $350 \mathrm{msnm}$; precipitación $923.5 \mathrm{~mm}$ ) aunque no coincide en los meses (enero-marzo) en que se presenta la temporada seca en el trabajo de Gómez-Restrepo (2010) para Hura crepitans L.

Tabla 3. Prueba de razón de verosimilitud para categorias diamétricas de Hura polyandra.

\begin{tabular}{cccc}
\hline Categorías & $R V$ & Gl & $P$ \\
\hline 5 y 10 & 2.5593 & 3 & 0.465 \\
5 y 15 & 4.7380 & 3 & 0.192 \\
5 y 20 & $0.0070 \mid$ & 3 & 1.000 \\
5 y 25 & 4.6791 & 3 & 0.197 \\
5 y 30 & 5.4443 & 3 & 0.142 \\
10 y 15 & 0.3231 & 3 & 0.956 \\
10 y 20 & 3.0019 & 3 & 0.391 \\
10 y 25 & 14.089 & 3 & 0.003 \\
10 y 30 & 13.032 & 3 & 0.005 \\
15 y 20 & 5.2821 & 3 & 0.152 \\
15 y 25 & 18.062 & 3 & 0.000 \\
15 y 30 & 16.053 & 3 & 0.001 \\
20 y 25 & 9.6594 & 3 & 0.022 \\
20 y 30 & 11.646 & 3 & 0.009 \\
25 y 30 & 0.7158 & 3 & 0.869 \\
\hline$R V:$ Razón de Verosimilitud, Gl: Grados de libertad, P: Probabilidad \\
$(P \leq 01)$
\end{tabular}




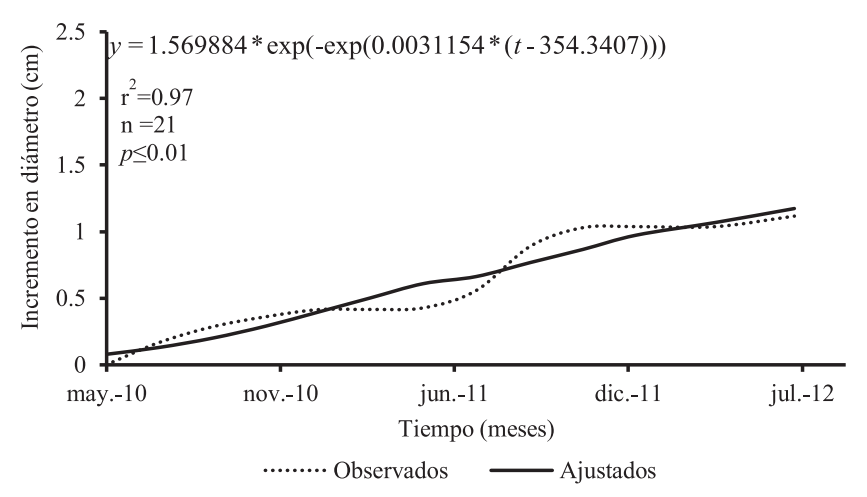

Figura 6. Resultados del ajuste de datos de crecimiento en diámetro para todos los árboles de Hura polyandra.
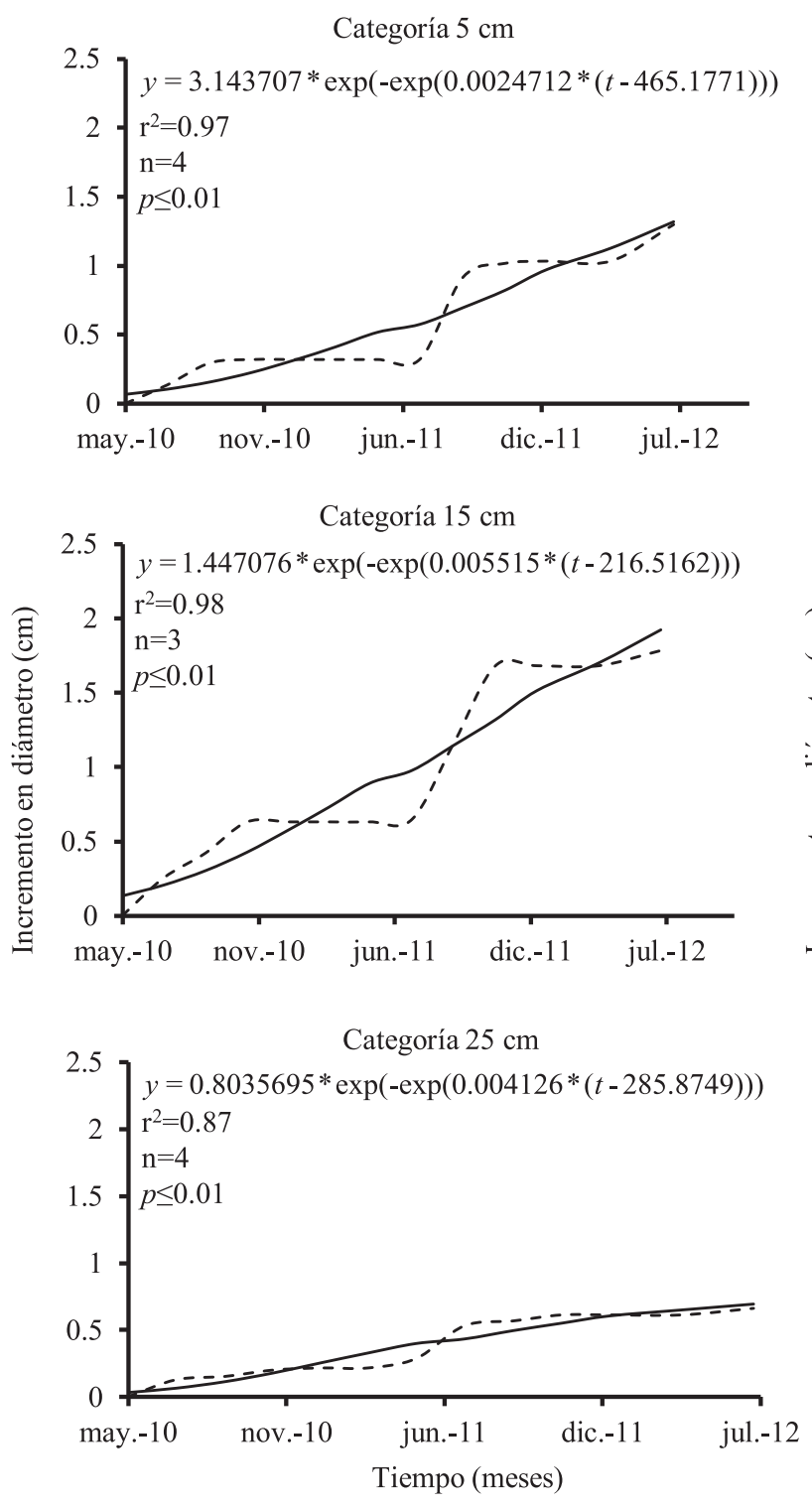

---- Observados — Ajustados
Tabla 4. Crecimiento promedio anual mínimo, medio y máximo de Hura polyandra.

\begin{tabular}{|c|c|c|c|c|c|}
\hline \multirow[t]{2}{*}{ Simetría } & \multirow[t]{2}{*}{$\begin{array}{l}\text { Número } \\
\text { de árboles }\end{array}$} & \multicolumn{3}{|c|}{$\begin{array}{l}\text { Crecimiento promedio } \\
\text { anual (cm/año) }\end{array}$} & \multirow[t]{2}{*}{$\begin{array}{l}\text { Desviación } \\
\text { estándar }\end{array}$} \\
\hline & & Mínimo & Medio & Máximo & \\
\hline 2 & 14 & 0.16 & 0.30 & 0.55 & 0.19 \\
\hline 3 & 7 & 0.18 & 0.26 & 0.53 & 0.18 \\
\hline $\begin{array}{l}\text { Todos } \\
\text { Iluminaciór }\end{array}$ & 21 & 0.17 & 0.28 & 0.54 & 0.18 \\
\hline 2 & 13 & 0.15 & 0.22 & 0.42 & 0.14 \\
\hline 3 & 8 & 0.19 & 0.36 & 0.70 & 0.25 \\
\hline Todos & 21 & 0.17 & 0.29 & 0.56 & 0.19 \\
\hline
\end{tabular}
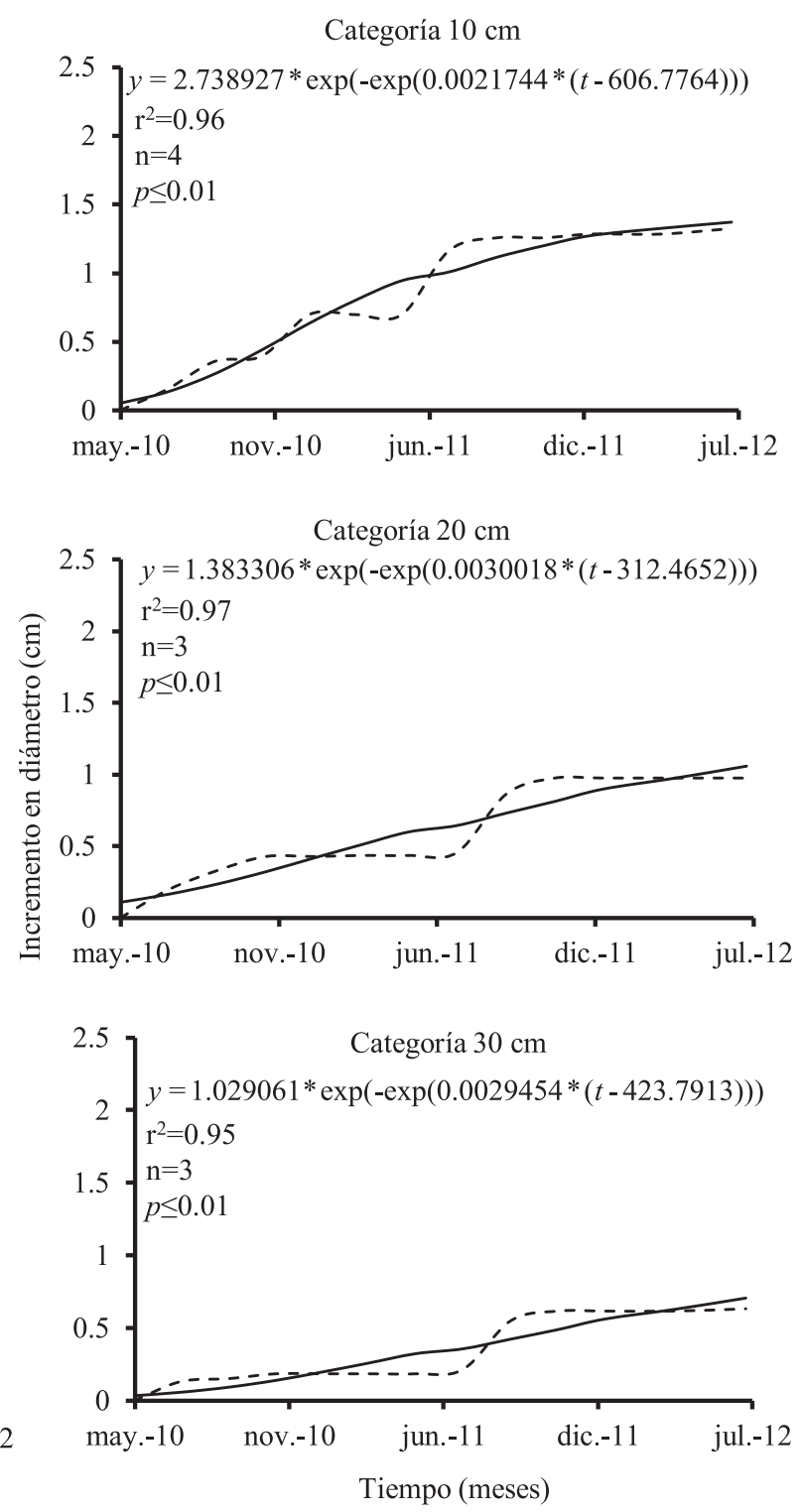

---- Observados — Ajustados

Figura 7. Crecimiento en diámetro para las categorías diamétricas de Hura polyandra. 

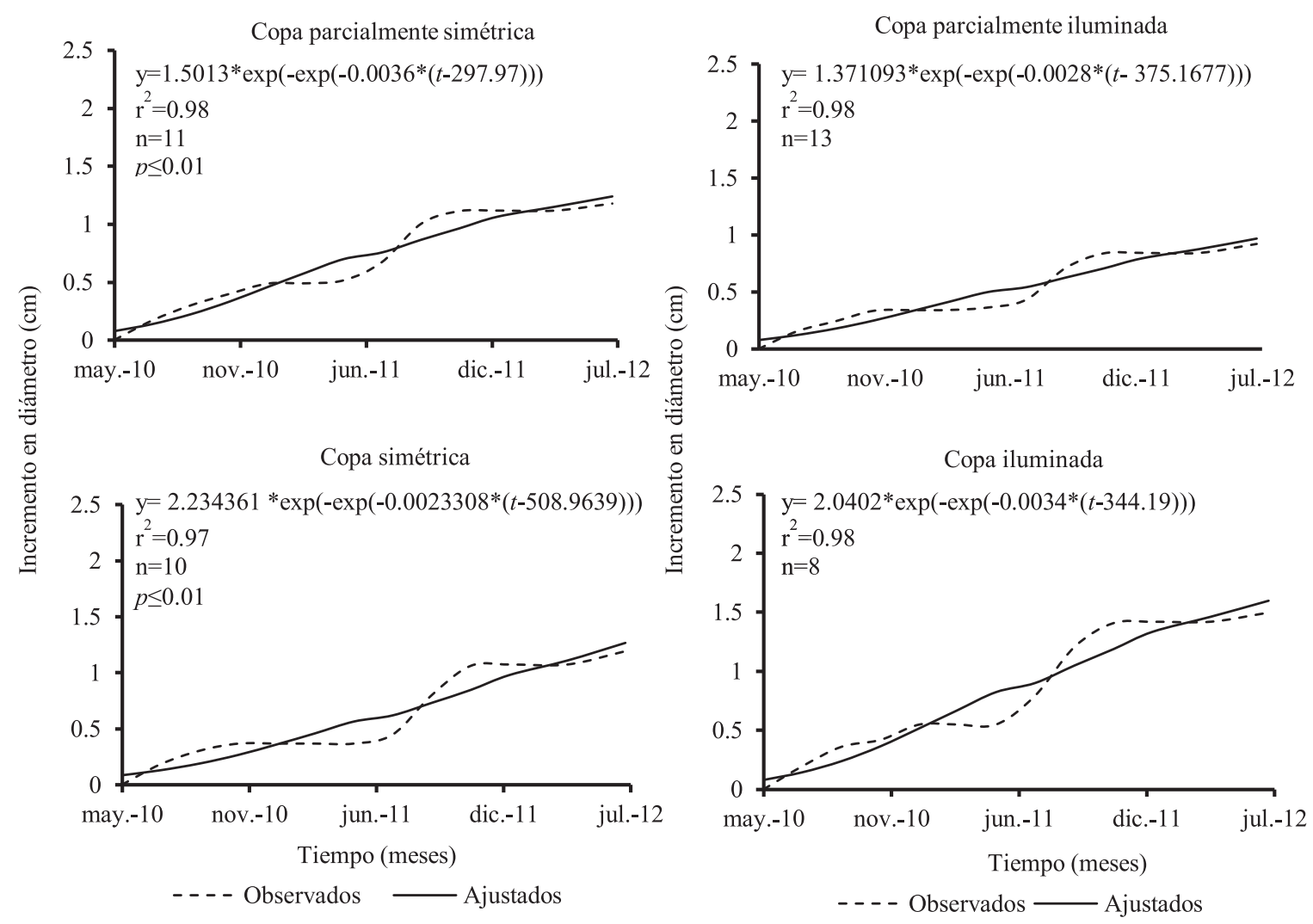

Figura 8. Crecimiento de árboles de Hura polyandra para simetrías e iluminaciones de copas.

Tabla 5. Prueba de razón de verosimilitud para condiciones de copa de Hura polyandra.

\begin{tabular}{|c|c|c|c|}
\hline Condiciones & $R V$ & Gl & $P$ \\
\hline \multicolumn{4}{|c|}{ Simetría de copa } \\
\hline 2 y 3 & 0.7064 & 3 & 0.872 \\
\hline \multicolumn{4}{|c|}{ Iluminación de copa } \\
\hline 2 y 3 & 15.402 & 3 & 0.002 \\
\hline
\end{tabular}

$R V$ : Razón de Verosimilitud, Gl: Grados de libertad, $P$ : Probabilidad $(P \leq 0.01)$

en Antioquía, Colombia (altitud: 530 - 545 m s.n.m.; precipitación: $1500-2500 \mathrm{~mm}$ ). En el anterior estudio el brote de hojas es en marzo y abril en la época de lluvias en Antioquia (Gómez-Restrepo, 2010) y en Sinaloa (Estrada-Castelo et al., 2012) coincide totalmente la temporalidad y el evento con el presente estudio.

En este trabajo Hura polyandra se defolió y floreció en respuesta al estrés hídrico de la temporada de secas, la disminución de humedad edáfica induce la caída de hojas con lo que disminuye la transpiración y por lo tanto se minimiza la perdida de agua, estrategia que además asegura la integridad del sistema conductor de los árboles de selvas secas (Mendez-Alonso et al., 2013).

El rebrote de hojas en esta especie tiene una explicación multifactorial, ya que no únicamente se relaciona con la estacionalidad de la lluvia, sino también con fenómenos meteorológicos ocasionales (ondas frías de alta humedad provenientes del océano pacífico) en los meses de diciembre, enero y febrero (Meza y García, 1997), la textura arcillosa del suelo (Meza y García, 1997) y su participación en el almacenamiento de humedad edáfica (Estrada-Castelo $e t$ al., 2012).

La floración de Hura polyandra en el Chilcahuite en marzo-mayo no coincide temporalmente con lo señalado por Goméz (2010), en cuyo estudio H. crepitans florece hasta en dos ocasiones durante la época de lluvias y por lo tanto se producen dos periodos de fructificación al año. En el presente estudio solo florece una al año durante la época seca relacionado con la estimulación hormonal (aumento de auxinas) por aumento gradual de la temperatura en primavera y la participación del fotoperiodo (Wright y Van Schaik, 1994).

La fructificación en esta especie se definió por su síndrome dispersión autocórica, que se ha observado en otras Euphorbiaceae como Hura crepitans y Croton magdalenensis Müll. Arg. ya que su dehiscencia puede llevarse a cabo en época de secas y en época de lluvias (Gómez-Restrepo, 2010), sin ajustarse estrictamente a la estacionalidad de la precipitación o a las oscilaciones de temperatura.

Se encontró una relación entre el diámetro de los árboles y la fenología foliar de Hura polyandra, que obedecería a 


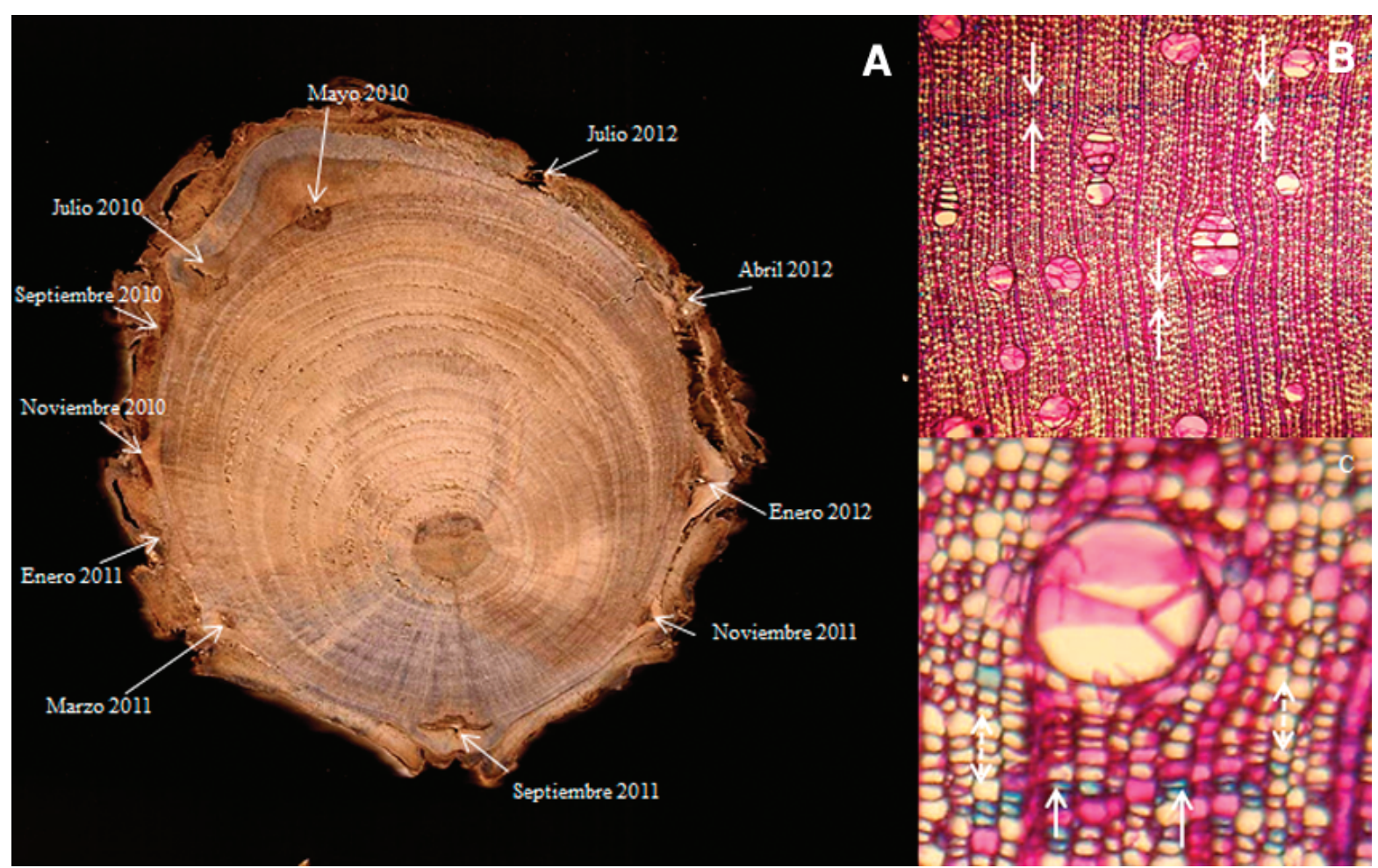

Figura 9. Periodicidad del crecimiento de Hura polyandra A) cicatrices en rodaja de $15 \mathrm{~cm}$. B) delimitación de anillos de crecimiento (flechas) (5x). C) bandas de parénquima (flechas discontinuas) e hilera de fibras (flechas continuas) $(20 \mathrm{x})$.

una mayor capacitación radical de árboles más viejos para aprovechar agua edáfica a mayores profundidades y que les permitirían conservar follaje la mayor parte del año (Estrada-Castelo et al., 2012). La fenología reproductiva y el diámetro presentaría dos vertientes: la floración no se relaciona con esta variable, por lo que depende de factores abióticos o endógenos (Lobo et al., 2003) y la mayor fructificación en árboles maduros se ha calificado como un buen indicador del potencial para proveer mayor abundancia de frutos (Chapman et al., 1992).

El crecimiento en diámetro promedio anual registrado en éste trabajo $(0.29 \mathrm{~cm})$ fue menor a lo estudiado por Luna (1994) en Venezuela para Hura crepitans $(0.37-0.40 \mathrm{~cm})$ en selvas con marcada estación seca. La estacionalidad de la precipitación es una de las variables que determina las diferencias entre tasas de crecimiento, aunque cada especie presenta atributos funcionales que modulan esta respuesta como: intensidad y duración de su brote de hojas, tasa de asimilación de carbono o de fotosíntesis (Lisi et al., 2008; Rossato, 2009).

Mora et al. (2009) en Venezuela demostraron que las tasas de crecimiento de las especies pioneras en categorías diamétricas menores a $30 \mathrm{~cm}$ pueden tener las más altas tasas de crecimiento. Cuando no hay un dosel completamente cerrado que impida el acceso de luz, las plántulas y árboles de categorías menores aprovechan este recurso creciendo en altura y diámetro al máximo, dadas las altas tasas de respira- ción y fotosíntesis que caracterizan a las pioneras (Binkley et al., 2002).

$\mathrm{Al}$ ser una especie que pondera la luz en sus copas para crecer, en selvas secas esto cobra relevancia por la periodicidad de los eventos fenológicos que las caracterizan ya que requieren tener una nueva producción de hojas para contribuir a la activación del cambium vascular y con ello al engrosamiento del tronco aprovechando la mayor cantidad de horas-luz y la estación lluviosa (Wright y Van Schaik, 1994).

Con los resultados de esta investigación se puede afirmar que Hura polyandra se comporta como una pionera demandante de luz que aprovecha además las condiciones edáfoclimáticas para crecer y que le permitirán iniciar sus eventos fenológicos reproductivos del siguiente año en plena estación seca (Wright y Van Schaik, 1994; Chaturvedi et al., 2011).

Periodicidad. Hura polyandra presentó anillos conspicuos, lo que se había encontrado en otra localidad del mismo municipio (Pineda-Herrera et al., 2012c) y difiere con lo investigado para $H$. crepitans en Sudamérica por Menegga (2005) en selvas con regímenes de precipitación mayor y donde los anillos no fueron distinguibles. En árboles tropicales se reconoce que la formación periódica de los anillos se relaciona con la presencia/ausencia de temporadas de lluvia o sequía (Worbes, 1999).

La periodicidad anual demarcada anatómicamente por 
Fenología, crecimiento en diámetro y periodicidad de Hura polyandra en Costa Grande, Guerrero, México.

parénquima y fibras coincide parcialmente con lo descrito para Hura crepitans en Perú por Beltrán-Gutiérrez y Valencia-Ramos (2013) donde únicamente se cita al parénquima como carácter delimitante. La gran cantidad de parénquima encontrado describe que esta especie almacena significativas reservas residuales (carbohidratos) producto de la actividad fotosintética del ciclo anterior y que no fueron utilizados en la fenología foliar o reproductiva (Kozlowski, 1992), mientras que las fibras complementan la estructura dando rigidez a la madera (Pineda-Herrera et al., 2012c).

Los cambios en la morfología y tamaño de las células durante la caída de hojas, es consecuencia de una disminución sustancial de la actividad cambial, al presentarse las lluvias los productos de la fotosíntesis elaborados con un pleno brote de hojas están disponibles para la reactivación del cambium y la consecuente formación de nuevas células del xilema (Beltrán-Gutiérrez y Valencia-Ramos, 2013). La porosidad difusa en Hura polyandra resalta como un carácter común en la mayoría de los miembros de la subfamilia Euphorbiaideae a la que pertenece el género Hura (Menegga, 2005) y su frecuencia también se ha relacionado con climas estacionales, donde la precipitación es el principal factor limitante (Beltrán-Gutiérrez y Valencia-Ramos, 2013).

\section{Conclusiones}

Hura polyandra presenta un patrón fenológico estacional. Su fenología foliar se asocia a la dinámica de la precipitación local y su intensidad se relaciona con árboles jóvenes. La fenología reproductiva se asocia al comportamiento de la temperatura y se ve favorecida en los árboles de mayor edad. Crece anualmente favorecida por la precipitación y las tasas de sus categorías diamétricas la caracterizan como una especie pionera que incrementa su diámetro favorablemente con copas totalmente iluminadas.

\section{Agradecimientos}

A Ismael Magaña ( $\dagger$ ) por el apoyo y las facilidades prestadas para el estudio, a la familia Bustos Herrera por su apoyo en campo, a los contribuyentes que través del CONACYT apoyaron económicamente al primer autor para sus estudios de posgrado, a los revisores asignados por Botanical Sciences y a Jorge Santana del Herbario Metropolitano UAMIZ Dr. Ramón Riva y Nava identificó e integró ejemplares botánicos de la especie.

\section{Literatura citada}

Baker T.R., Affum-Baffoe K., Burslem D.F.R.P. y Swaine M.D. 2002. Phenological differences in tree water use and the timing of tropical forest inventories: conclusions from patterns of dry season diameter change. Forest Ecology and Management 171:261-274.
Baker T.R., Swaine M.D. y Burslem D.F.R.P. 2003. Variation in tropical forest growth rates: combined effects of functional group composition and resource availability. Perspectives in Plant Ecology, Evolution and Systematics 6:21-36.

Beltrán-Gutiérrez L.A. y Valencia-Ramos G.M. 2013. Anatomía de anillos de crecimiento de 80 especies arbóreas potenciales para estudios dendrocronológicos en la Selva Central, Perú. Revista de Biología Tropical 61:1025-1037.

Binkley D., Stape J.L., Ryan M.G., Barnard H.R. y Fownes J. 2002. Age-related decline in forest ecosystem growth: An IndividualTree, Stand-Structure Hypothesis. Ecosystems 5:58-67.

Borchert R. 1994. Soil and stem water storage determine phenology and distribution of tropical dry forest trees. Ecology 75:1437-1449.

Borchert R., Meyer S.A., Felger R.S. y Porter-Bolland L. 2004. Environmental control of flowering periodicity in Costa Rican and Mexican tropical dry forests. Global Ecology and Biogeography 13:409-425.

Bullock S.H. y Solis-Magallanes J.A. 1990. Phenology of canopy trees of a tropical deciduous forest in Mexico. Biotropica 22:22-35.

Bullock S.H. 1997. Effects of seasonal rainfall on radial growth in two tropical tree species. International Journal of Biometeoro$\log 4$ 41:13-16.

Bullock S.H. 2002. La fenología de plantas en Chamela. En: Noguera, F.A., Vega-Rivera J.H., García-Aldrete A.N. y QuesadaAvendaño M. Eds. Historia Natural de Chamela, pp 491-498, Universidad Nacional Autónoma de México, México, D.F.

Ceccon E., Huante P., y Rincón E. 2006. Abiotic factors influencing tropical dry forests regeneration. Brazilian Archives of Biology and Technology 49:305-312.

Chapman C.A. y Chapman L.J. 1990. Density and growth rate of some tropical dry forest trees: Comparisons between succesional forest types. Bulletin of the Torrey Botanical Club 117:226-231.

Chapman C.A., Chapman L.J., Wangham R., Hunt K., Gebo D. y Gardner L. 1992. Estimators of fruit abundance of tropical trees. Biotropica 24:527-531.

Chaturvedi R.K., Raghubanshi A.S. y Singh J.S. 2011. Leaf attributes and tree growth in a tropical dry forest. Journal of Vegetation Science 22:917-931.

CONABIO [Comisión Nacional para el Conocimiento y Uso de la Biodiversidad] 2011. Fichas de especies prioritarias. Guacamaya Verde (Ara militaris). Comisión Nacional de Áreas Naturales Protegidas/Comisión Nacional para el Conocimiento y Uso de la Biodiversidad, México D.F.

Dawkins H.C. 1958. Crown diameters: their relation to bole diameter in tropical forest trees. Commonwealth Forestry Review 42:318-333.

Detienne P. 1989. Appearance and periodicity of growth rings in some tropical woods. IAWA Journal 10:123-132.

Detienne P. 1995. Nature et périodicité des cernes dans quelques bois guyanais. Bois et Foréts des Tropiques 243:65-75.

Estrada-Castelo J.A., Marquez G. y Higareda C. 2012. Fenologia Foliar de Diez Especies Arboreas. En el mineral de Nuestra Señora de la Candelaria, Cosalá, Sinaloa. Editorial Académica Española. Saarbrücken.

Flores J.S. y Ricalde R.V. 1996. The secretions and exudates of plants used in Mayan traditional medicine. Journal of Herbs, Spices and Medicinal Plants 4:53-59. 
Fournier-O L.A. y Charpantier C. 1975. El tamaño de la muestra y la frecuencia de las observaciones en el estudio de las características fenológicas de los árboles tropicales. Turrialba 25:45-48.

Galán-Larrea R., Valdez-Hernández J.I., De los Santos-Posadas H.M. y López-Ayala J.L. 2011. Periodicidad en la madera de cuatro especies arbóreas de la costa de Oaxaca. En: A. R. Endara-Agramont A.R., Mora-Santacruz A. y Valdez-Hernández J.I. Eds. Bosques y Árboles del Trópico Mexicano: Estructura, Crecimiento y Usos, pp. 37-56, Universidad de Guadalajara/ Prometeo Editores, Guadalajara.

Gallardo C. 1996. Parque ecológico La Vainilla. Zihuatanejo, Guerrero. Estudios florísticos de Guerrero. No. 8. Facultad de Ciencias-Universidad Nacional Autónoma de México, México D.F.

Gómez-Restrepo M.L. 2010. Fenología reproductiva de especies forestales nativas presentes en la jurisdicción de Corantioquia. Un paso hacia su conservación. Vol. 1. Corporación Autónoma Regional del Centro de Antioquia, Medellín, Colombia. http:// www.corantioquia.gov.co/sitios/ExtranetCorantioquia/SiteAssets/Lists/Administrar\%20Contenidos/EditForm/fenologia. pdf\#search=Fenolog $\%$ C3\%ADa\%20reproductiva $\% 20 d e \% 20$ especies $\% 20$ forestales $\% 20$ nativas $\% 20$ presentes $\% 20$ en $\% 201$ a\%20jurisdicci\%C3\%B3n\%20de\%20Corantioquia. Consultado el 16 de Marzo de 2104.

Gotelli N.J. y Ellison A.M. 2004. A primer of ecological statistics. Sinauer Associates, Sunderland, Massachusetts.

Hayden B., Greene D.F. y Quesada M. 2010. A field experiment to determine the effect of dry-season precipitation on annual ring formation and leaf phenology in a seasonally dry tropical forest. Journal of Tropical Ecology 26:237-242.

Herrerías-Diego Y., Quesada M., Stoner K.E. y Lobo, J.A. 2006. Effects of forest fragmentation on phenological patterns and reproductive success of the tropical dry forest tree Ceiba aesculifolia. Conservation Biology 20:1111-1120.

INEGI [Instituto Nacional de Estadística Geografía e Informática] 2005. Carta de uso del suelo y vegetación: escala 1:250 000. Serie III Instituto Nacional de Estadística, Geografía e Informática, Aguascalientes.

Justiniano M.J. y Fredericksen T.S. 2000. Phenology of tree species in Bolivian dry forests. Biotropica 32:276-281.

Kimura D.K. 1980. Likelihood methods for the Von Bertalanffy growth curve. Fishering Bulletin 77:765-776.

Kinnaird M.F. 1992. Phenology of flowering and fruiting of an East African riverine forest ecosystem. Biotropica 24:187-194.

Kozlowski T. 1992. Carbohydrate source and sinks in woody plants. The Botanical Review 58:107-222. http://www.jstor.org/ stable/4354186?seq=1\#page_scan_tab_contents. Consultado el 15 de Febrero de 2104.

Lisi C.S., Tomazello M., Botosso P.C., Roig F.A., Maria V.R.B., Ferreira-Fedele L., y Voigt A.R.A. 2008. Tree-ring formation, radial increment periodicity, and phenology of tree species from a seasonal semi-deciduous forest in southeast Brazil. Iawa Journal 29:189-207.

Lobo J.A., Quesada M., Stoner K.E., Fuchs E.J., Herrerías-Diego Y., Rojas J. y Saborío G. 2003. Factors affecting phenological patterns of bombacaceous trees in seasonal forests in Costa Rica and Mexico. American Journal of Botany 90:1054-1063.

López-Ayala J.L., Valdez-Hernández J.I., Terrazas T. y Valdez-Lazalde R. 2006a. Crecimiento en diámetro de especies arbóreas en una selva mediana subcaducifolia en Colima, México. Agrociencia 40:139-147.

López-Ayala J.L., Valdez-Hernández J.I., Terrazas T. y Valdez-Lazalde R. 2006b. Anillos de crecimiento y su periodicidad en tres especies tropicales del estado de Colima, México. Agrociencia 40:533-544.

López L., Villalba R. y Peña-Claros M. 2012. Ritmos de crecimiento diamétrico en los bosques secos tropicales: aportes al manejo sostenible de los bosques de la provincia biogeográfica del Cerrado Boliviano. Bosque (Valdivia) 33:211-219

Luna A. 1994. Estudio sobre el crecimiento y edad de 20 especies forestales de los bosques naturales venezolanos Parte I. FUNDACITE-IFLE-Universidad de los Andes, Mérida, Venezuela.

Makocki M., Valdez-Hernández J.I. y Gracía-Moya E. 2012. Crecimiento de tres especies arbóreas en una selva mediana subcaducifolia de Nayarit. En: Salcedo-Pérez E., Hernández-Álvarez E., Vázquez-Gracía J.A., Escoto-García T. y Díaz-Echeverría N. Eds. Recursos forestales en el occidente de México: Diversidad, Manejo, Producción, Aprovechamiento y Conservación, pp. 182-206. Universidad de Guadalajara-Amaya ediciones, Guadalajara.

Marques M.C.M., Roper J.J. y Baggio-Salvalaggio A.P. 2004. Phenological patterns among plant life-forms in a subtropical forest in southern Brazil. Plant Ecology 173:203-213.

Melo-Cruz O.A. y Vargas-Ríos R. 2003. Evaluación ecológica y silvicultural de ecosistemas boscosos. Universidad del Tolima/ CRQ/CORPOCALDAS/CORTOLIMA, Ibagué.

Méndez-Alonzo R., Pineda-García F., Paz H., Rosell J.A. y Olson M.E. 2013. Leaf phenology is associated with soil water availability and xylem traits in a tropical dry forest. Trees 27:745-754

Mendivelso H.A., Camarero J.J., Gutiérrez E. y Zuidema P.A. 2014. Time-dependent effects of climate and drought on tree growth in a Neotropical dry forest: Short-term tolerance $v s$. long-term sensitivity. Agricultural and Forest Meteorology 188:13-23.

Mennega A. 2005. Wood anatomy of the subfamily Euphorbioideae. A comparison with subfamilies Crotonoideae and Acalyphoideae and the implications for the circumscription of the Euphorbiaceae. IAWA Journal 26:1-68.

Meza L. y García J.L. 1997. Vegetación y mesoclima de Guerrero. Estudios florísticos de Guerrero Núm. Especial. Facultad de Ciencias-UNAM, México, D.F.

Miranda F. y Hernández-X. E. 1963. Los tipos de vegetación de México y su clasificación. Boletín de la Sociedad Botánica de México 28:29-179.

Mittermeier K.A., Myers N., Robles-Gil P. y Goettsch-Mittermeier C. 1999. Biodiversidad amenazada: Las Ecorregiones Terrestres Prioritarias del Mundo. Cemex/Agrupación Sierra Madre S.C./Conservación Internacional. México, D.F.

Mora E.M., Ramírez-Angulo H., y Torres-Lezama A. 2009. Análisis de las tasas de crecimiento diametral por grupos funcionales de especies arbóreas en un bosque tropical semi-decíduo de Venezuela. Revista Forestal Venezolana 53:23-31

Murali K.S. y Sukumar R. 1994. Reproductive phenology of a tropical dry forest in Mudumalai, southern India. Journal of Ecology 82:759-767.

Myers B.A., Williams R.J., Fordyce I., Duff G.A. y Eamus D. 1998. Does irrigation affect leaf phenology in deciduous and evergreen trees of the savannas of northern Australia? Australian Journal of Ecology 23:329-339. 
Pennington T.D. y Sarukhán J. 2005. Árboles tropicales de México: Manual para la identificación de las principales especies. Fondo de Cultura Económica/Universidad Nacional Autónoma de México, México, D. F.

Pineda-Herrera E., de la Paz-Pérez-Olvera C. y Valdez-Hernández J.I. 2012a. El aprovechamiento maderable en Costa Grande, Guerrero. Biodiversitas 102:6-11.

Pineda-Herrera E., Valdez-Hernández J.I. y López-López M.A. 2012b. Fenología de Schizolobium parahyba y Vochysia guatemalensis en una selva alta perennifolia de Oaxaca, México. Botanical Sciences 90:185-193.

Pineda-Herrera E, de la Paz-Pérez-Olvera C., Dávalos-Sotelo R. y Valdez-Hernández J.I. 2012c. Características tecnológicas de la madera de dos especies de Costa Grande, Guerrero, México. Madera y bosques 18:53-71.

Román M.L., Mora S.A. y Gallegos R.A. 2011. Árboles tropicales de uso múltiple en la Costa de Jalisco, México, En: EndaraAgramont A.R., Mora-Santacruz A y Valdez-Hernández J.I. Eds. Bosques y Árboles del Trópico Mexicano: Estructura, Crecimiento y Usos, pp. 81-106, Universidad de Guadalajara/ Prometeo Editores, Guadalajara.

Rossatto D.R. 2009. Branch elongation and diameter growth were temporally dissociated in "cerrado" tree species. Hoehnea 36:437-444

Rzedowski J. 1978. Vegetación de México. Limusa, México, D.F.

Rzedowski J. 1991. Diversidad y orígenes de la flora fanerogámica de México. Acta Botanica Mexicana 14: 3-21.

Salgado-Ugarte I.H., Gómez-Márquez J.L. y Peña-Mendoza B. 2005. Métodos actualizados para análisis de datos biológicopesqueros. Universidad Nacional Autónoma de México-Facultad de Estudios Superiores Zaragoza, México, D.F.

Sandoval E. 2005. Técnicas aplicadas al estudio de la anatomía vegetal. Cuadernos del Instituto de Biología 38. Universidad Nacional Autónoma de México, México, D.F.
Shackleton C.M. 2002. Growth patterns of Pterocarpus angolensis in savannas of the South African lowveld. Forest Ecology and Management 166:85-97.

Silva J.A. 2008. Fichas técnicas sobre características tecnológicas y usos de maderas comercializadas en México. Tomo 1. CONAFOR. Zapopan.

SMN [Servicio Meteorológico Nacional]. 2012. Datos de precipitación y temperatura de la estación automática Vallecitos de Zaragoza. Agosto de 2012.

Sokpon N. y Biaou S.H. 2002. The use of diameter distributions in sustained-use management of remnant forests in Benin: case of Bassila forest reserve in North Benin. Forest Ecology and management 161:13-25.

StataCorp. 2001. Stata Statistical Software: Release 7.0. Stata corporation, College Station, Texas.

Valdez-Hernández M., Andrade J.L., Jackson P.C. y RebolledoVieyra M. 2010. Phenology of five tree species of a tropical dry forest in Yucatan, Mexico: effects of environmental and physiological factors. Plant and soil 329:155-171.

Yañez-Espinosa L., Terrazas T. y Lopez-Mata L. 2006. Integrated analysis of tropical trees growth: a multivariate approach. Annals of botany 98:637-645.

Whigham D.F., Zugasty-Towle P., Cabrera-Cano E., O’Neill J. y Ley E. 1990. The effect of annual variation in precipitation on growth and litter production in a tropical dry forest in the Yucatan of Mexico. Tropical Ecology 31:23-34.

Williams R.J., Myers B.A., Muller W.J., Duff G.A. y Eamus D. 1997. Leaf phenology of woody species in a north Australian tropical savanna. Ecology 78:2542-2558.

Worbes M. 1999. Annual growth rings, rainfall-dependent growth and long-term growth patterns of tropical trees from the Caparo Forest Reserve in Venezuela. Journal of ecology 87:391-403.

Wright S.J. y Van Schaik C.P. 1994. Light and the phenology of tropical trees. American Naturalist 143:192-199.

Recibido: 16 de abril de 2014

Aceptado: 3 de septiembre de 2014 